\title{
Révision de la formation complémentaire en médecine manuelle
}

\author{
Dès le $1^{\text {er }}$ janvier 2013, le Certificat de capacité «Médecine manuelle SAMM» sera \\ délivré en conformité avec les critères du Système de Bologne. Cette formation \\ complémentaire de deux ans, que le/la candidat(e) suit en emploi, lui donne une \\ compétence accrue et une meilleure autonomie dans les applications diagnostiques \\ et thérapeutiques de la médecine manuelle.
}

Ulrich W. Böhni

Président de la SAMM

Correspondance: Dr Ulrich W. Böhni Société Suisse de Médecine Manuelle SAMM c/o Mediapolis AG Röschstrasse 18 CH-9006 St-Gall Tél. 0712465181

info[at]samm.ch www.samm.ch

\begin{abstract}
La Médecine Manuelle moderne est une méthode de traitement des troubles fonctionnels du système locomoteur basée sur les sciences naturelles. Elle consiste à soulager les douleurs et à améliorer le bien-être des patients par des manipulations ciblées. Durant cette formation complémentaire, le/la candidat(e) acquiert la capacité de procéder au diagnostic différencié et au traitement ciblé de douleurs articulaires, dorsales et musculaires. Depuis le $1^{\text {er }}$ janvier 1999, le Certificat de capacité «Médecine manuelle SAMM» de la FMH est délivré à l'issue de cette formation.
\end{abstract}

\section{Révision du Certificat de capacité}

Le comité directeur de la Société Suisse de Médecine Manuelle SAMM a soumis une demande de révision de ce certificat de capacité à l'ISFM/FMH dans le but d'adapter la formation complémentaire aux nouveaux critères de formation postgraduée universitaire. La formation complémentaire, qui était déjà dispensée sous forme de modules, a été restructurée de telle manière que les «Bases de la Médecine Manuelle SAMM» correspondent désormais à un Certificate of Advanced Studies (CAS) et le Certificat de capacité à un Diploma of Advanced Studies (DAS). Le processus de validation universitaire est en cours.

\section{Bases de la Médecine Manuelle SAMM}

Le/la candidat(e) qui a suivi les 3 modules de 4 jours des «Bases de la Médecine Manuelle SAMM» est capable de réaliser un examen fonctionnel clinique différencié de l'ensemble du système locomoteur, d'évaluer correctement les syndromes douloureux musculosquelettiques et de poser les indications d'une thérapie manuelle. S'y ajoute la capacité d'appliquer certaines techniques de mobilisation sans impulsion.

\section{Attestation de formation complémentaire}

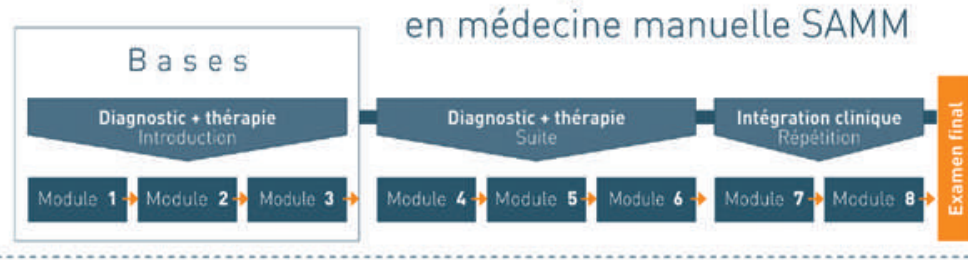

Certificat de capacité «Médecine Manuelle SAMM» Le/la candidat(e) au Certificat de capacité «Médecine manuelle SAMM» est astreint à suivre au total 8 modules de 4 jours et doit réussir les examens écrits et cliniques. Cette formation complémentaire complète, couplée à des activités en groupes d'apprentissage et à un travail personnel, permet d'acquérir la connaissance des syndromes douloureux régionaux du système musculo-squelettique ainsi que la capacité d'appliquer toutes les méthodes de la médecine manuelle - y compris la mobilisation avec impulsion - dans le diagnostic et le traitement. L'application pratique en milieu clinique à des cas-types de patients est également intégrée dans la formation.

\section{Examens}

Pour l'obtention du CAS, la réussite d'un examen écrit s'ajoute aux exigences de participation à l'enseignement et d'atteinte des objectifs didactiques. L'examen porte sur la matière enseignée dans les modules 1 à 3 et inclut des questions des deux branches de base que sont l'anatomie et la biomécanique. A l'issue du module 8 , les candidat(e)s au Certificat de capacité doivent réussir un autre examen écrit ainsi qu'un examen pratique de médecine manuelle.

\section{Formation pratique}

Les titulaires du Certificat de capacité de Médecine Manuelle SAMM proviennent de différentes disciplines. Les prestataires de soins de base (médecins de famille) y sont fortement représentés, mais de nombreux candidats nous viennent d'autres disciplines comme la rhumatologie, la réadaptation, l'orthopédie et le traitement de la douleur. Tous considèrent la formation en Médecine Manuelle comme un complément utile à la pratique quotidienne en cabinet ou à l'hôpital. Après tout, la Médecine Manuelle est une méthode de traitement intégrale peu coûteuse qui soulage les douleurs et produit des résultats tangibles chez le patient dans un délai rapide. A partir de l'année prochaine, cette formation complémentaire sera dispensée en conformité avec les critères de la nouvelle structure de formation postgraduée de Bologne. La première formation de deux ans selon le nouveau système démarrera le 9 janvier 2013. 